\title{
Construção de domínios do Event History Calendar: Elementos metodológicos da Teoria Fundamentada nos Dados construtivista
}

\author{
Jaqueline Silva Santos ${ }^{1}$, Jeniffer Stephanie Marques Hilário², Raquel \\ Dully Andrade ${ }^{3}$ e Débora Falleiros de Mello² \\ ${ }^{1}$ Superintendência Regional de Saúde de Passos, Brasil | jaque_fesp@hotmail.com | \\ https://orcid.org/0000-0002-7543-5522 \\ ${ }^{2}$ Escola de Enfermagem de Ribeirão Preto/Universidade de São Paulo, Brasil \\ jsmhilario@outlook.com; defmello@eerp.usp.br | https://orcid.org/0000-0001-5541-6546; \\ https://orcid.org/0000-0001-5359-9780 \\ 3Universidade do Estado de Minas Gerais - Campus Passos, Brasil | radully@gmail.com | \\ https://orcid.org/0000-0002-1515-098X
}

Resumo: Introdução: O Event History Calendar (EHC) é uma ferramenta de cuidado à saúde, estruturada em colunas e linhas. As linhas refletem os domínios dessa ferramenta e podem ser traduzidas em atividades, comportamentos e/ou eventos de vida do sujeito. Objetivo: Apresentar elementos metodológicos envolvidos no processo de construção dos domínios da ferramenta EHC para mães adolescentes. Métodos: Trata-se de um estudo qualitativo, realizado em um município brasileiro de médio porte, com 11 mães adolescentes que tinham crianças menores de dois anos de idade. O processo de coleta dos dados e a construção dos domínios da ferramenta EHC foi pautado em elementos da Teoria Fundamentada nos Dados (TFD) vertente construtivista. Na coleta de dados, foi utilizada a técnica do snowball associada à amostragem teórica. Foram construídas seis cadeias de referência e três grupos amostrais de mães adolescentes. Nesse processo analítico foram utilizadas a codificação inicial linha a linha, a codificação focalizada, o método comparativo constante, a redação de memorandos, a elaboração de diagramas e a amostragem teórica. Foram construídas cinco categorias centrais e vinte subcategorias. Resultados: $\mathrm{Na}$ elaboração da proposta de ferramenta, os domínios do EHC foram construídos por meio da análise das falas das mães adolescentes que apontaram aspectos sobre o cuidado de si e 0 cuidado da criança, bem como os fatores envolvidos nos processos de cuidado, com destaque para a rede de apoio, as vulnerabilidades, as situações e emoções vivenciadas. Conclusões: Elementos metodológicos da TFD construtivista forneceram alicerces para a construção dos domínios da ferramenta EHC para mães adolescentes.

Palavras-chave: Saúde da Criança; Saúde do Adolescente; Cuidados de Enfermagem; Pesquisa Qualitativa; Teoria Fundamentada.

\section{Building Event History Calendar Domains: Methodological Elements of Constructivist} Grounded Theory

Abstract: Introduction: Event History Calendar (EHC) is a healthcare tool, arranged in columns and rows; the latter reflect the domains of this tool and can be translated into activities, behaviors, and/or events in peoples' lives. Goal: To present methodological elements involved in the process of building EHC domains for adolescent mothers. Methods: This is a qualitative study conducted in a medium-sized Brazilian municipality with eleven adolescent mothers of children under two years of age. Data collection and EHC domain building were based on elements of the constructivist Grounded Theory (GT). Data collection used the snowbal technique associated with theoretical sampling. Six reference chains and three sample groups of adolescent mothers were constructed. This analytical process used initial line-by-line coding, focused coding, constant comparison, memo writing, diagram making, and theoretical sampling. Five core categories and 20 subcategories were built. Results: When designing the tool proposal, we built the EHC domains by analyzing the adolescent mothers' speeches that revealed aspects about the care of the self and the care of the child, as well as factors involved in the care processes, especially support network, vulnerabilities, experienced situations and emotions. Conclusion: Methodological elements of constructivist GT provided a foundation to build EHC domains for adolescent mothers.

Keywords: Child Health; Adolescent Health; Nursing Care; Qualitative Research; Grounded Theory. 


\section{Introdução}

O cuidado voltado a promoção da saúde da adolescente pode aparecer como um desafio para o profissional enfermeiro (Santos et al., 2020), principalmente quando a adolescente se torna mãe, o que aponta a necessidade de ferramentas que apoiam o estabelecimento do processo comunicativo (Munro-Kramer et al., 2017).

Nesse cenário, emerge as potencialidades do Event History Calendar (EHC), ferramenta para a coleta de dados retrospectivos referentes à ocorrência e sequência de eventos, relacionados a períodos curtos e longos (Martyn \& Belli, 2002). Na estrutura do EHC, as colunas denotam as unidades temporais e as linhas apresentam os domínios, que podem ser traduzidos em eventos, comportamentos ou atividades (Martyn \& Belli, 2002).

Nos momentos de cuidado, os enfermeiros podem utilizar o EHC para auxiliar no processo comunicativo com o adolescente e apoiar o levantamento de histórias contextualizadas (Martyn et al., 2012). Ao trazer possíveis contribuições para o processo dialógico contextualizado, a utilização do EHC pode ampliar a atuação do enfermeiro com a mãe adolescente (Santos et al., 2021).

Assim, com os domínios do EHC podem ser levantados dados de diferentes dimensões de vida, como comportamentos, atividades realizadas, eventos e transições vivenciados (Martyn \& Belli, 2002), em busca de uma abordagem centrada no sujeito (Munro-Kramer et al., 2017). Nesse sentido, emergiu a questão norteadora do estudo: "Quais elementos metodológicos podem ser utilizados para a construção dos domínios da ferramenta EHC?".

O objetivo do estudo foi apresentar elementos metodológicos envolvidos no processo de construção dos domínios da ferramenta EHC para mães adolescentes.

\section{Metodologia}

\subsection{Tipo de Estudo e Referencial Metodológico}

Estudo qualitativo em que o processo de coleta dos dados e construção dos domínios da ferramenta EHC foi pautado em elementos da Teoria Fundamentada nos Dados (TFD), vertente construtivista (Charmaz, 2009).

A TFD apresenta-se como uma linha metodológica que possibilita o estabelecimento de relações entre os fenômenos (Gabatz et al., 2017), abertura ao que ocorre em uma área substantiva, reconhecendo e nomeando os padrões de comportamento por meio de conceitos (Andrews et al., 2017). Os métodos da teoria fundamentada caracterizam-se pela flexibilidade e, na sua vertente construtivista, o entendimento é que a TFD é um modo de aprendizagem sobre os fenômenos estudados e também uma forma de os compreender (Charmaz, 2009).

$\mathrm{Na}$ presente pesquisa, os elementos da TFD construtivista utilizados foram: coleta de dados relevantes, buscando material sólido para uma análise significativa, e análise simultânea; codificação linha a linha inicial, que possibilita a análise minuciosa e rigorosa dos fragmentos dos dados, e codificação focalizada, momento em que o material dos códigos iniciais mais significativos ou frequentes é selecionado e contrastado com dados mais amplos; método comparativo constante, que permeia todo o processo analítico sendo a essência da análise da teoria fundamentada, pois a comparação constante dos dados, códigos e categorias possibilita uma compreensão conceitual e uma análise rigorosa, ao definir e abordar as propriedades analíticas das categorias; elaboração de memorandos, anotações analíticas escritas ao longo de toda a pesquisa, e diagramas, representações gráficas que buscam a integração e o estabelecimento da lógica de organização das ideias; e amostragem teórica, estratégia específica e sistemática utilizada para elaborar e refinar as categorias emergentes e para o alcance da variação (Charmaz, 2009). 
Cabe ressaltar que o objeto deste estudo não se direcionou para a construção de um modelo teórico, mas sim para a elaboração dos domínios de uma ferramenta, o EHC (Santos et al., 2021).

\subsection{Campo de Estudo e Participantes}

Estudo realizado em um município de médio porte do interior do estado de Minas Gerais, Brasil, com 11 mães adolescentes de crianças menores de dois anos de idade (Santos et al., 2021).

Foram estabelecidos como critérios de inclusão: idade entre 12 e 18 anos, filho(a) menor de dois anos, seguimento da saúde em Unidade de Saúde da Família (USF) e residência no município estudado (Santos et al., 2021). Os critérios de exclusão foram: interrupção do seguimento na USF, mudança de endereço fora das áreas de abrangência das USF do município, não ser encontrada após três tentativas realizadas por contato telefônico e/ou visita domiciliar (Santos et al., 2021).

Para a seleção das participantes e condução da coleta de dados foi utilizada a técnica do snowball (Biernacki \& Waldorf, 1981) associada à amostragem teórica (Charmaz, 2009). Não houve recusa para participação na pesquisa, as participantes foram denominadas E1, E2, E3...E11 (Santos et al., 2021).

\subsection{Coleta e Análise dos dados}

Os dados foram coletados de agosto a dezembro de 2017, com a utilização de um roteiro de entrevista com questões amplas e abertas para apreensão de particularidades e experiências das mães adolescentes (Santos et al., 2021). As entrevistas foram gravadas em áudio e tiveram duração média de 40 minutos, sendo realizadas pela primeira autora por meio de visita domiciliária (Santos et al., 2021)

Inicialmente foi realizado contato com integrantes das equipes de Saúde da Família (eSF), buscando auxilio na identificação e localização de mães adolescentes que atendiam os critérios de inclusão do estudo (Santos et al., 2021). As cadeias de referência foram iniciadas em locais com diferentes condições socioeconômicas do município (área central, área periférica e área periférica com condições socioeconômicas vulneráveis) (Santos et al., 2021). Foram construídas seis cadeias de referência e três grupos amostrais de mães adolescentes (Santos et al., 2021).

O primeiro grupo amostral foi constituído por três mães adolescentes (E1 ...E3) (Santos et al., 2021). Por meio dos relatos dessas participantes iniciou-se o processo de construção das categorias, o que possibilitou a realização de indagações sobre o processo de cuidar da criança e a identificação da necessidade de apreender situações do cuidado desempenhado por mães adolescentes em diferentes cenários vivenciados (Santos et al., 2021).

Para o processo de amostragem teórica, emergiu a necessidade de levantar especificidades e apreender experiências vivenciadas em diversas conjunturas, atreladas ao cuidado da criança em diferentes fases do desenvolvimento, ao cuidado de mais de um filho, a situações envolvendo a rede de apoio (fortalecida ou fragilizada). Assim, as cadeias de referências eram iniciadas para atender as questões que emergiam após realização e codificação de cada entrevista, tendo em vista a necessidade de continuidade no processo de desenvolvimento das propriedades das categorias e a busca por variação. O segundo grupo amostral foi constituído por seis mães adolescentes (E4... E9) e permitiu o levantamento das particularidades envolvidas no processo autocuidado da adolescente mãe e de cuidado da criança, em diferentes contextos vivenciados, com destaque para a presença e/ou ausência de uma rede de apoio e suas repercussões nas trajetórias de cuidado (Santos et al., 2021).

Em continuidade ao processo de refinamento das categorias, percebeu-se a necessidade de apreender duas situações: o planejamento da gestação e a participação em movimentos sociais. 
O terceiro grupo amostral foi constituído por duas mães adolescentes (E10 e E11) e apresentou os elementos necessários para o alcance da saturação teórica (Santos et al., 2021).

Os dados eram coletados e analisados de forma concomitante (Santos et al., 2021). Nesse processo analítico realizado pela primeira autora, foram utilizadas a codificação inicial linha a linha, a codificação focalizada, o método comparativo constante, a redação de memorandos, a elaboração diagramas e a amostragem teórica (Charmaz, 2009). No processo de codificação foram usadas palavras para denotar sentido de ação (Charmaz, 2009), pois o entendimento é que a adoção do gerúndio traduz a ideia de dinamicidade (Santos et al., 2016).

A Tabela 1 apresenta exemplos da codificação dos dados e construção de uma categoria (Santos, 2018).

Tabela 1. Exemplos da codificação dos dados e construção de uma categoria. Passos-MG, 2018.

\begin{tabular}{|c|c|c|}
\hline Exemplos de códigos & Subcategorias & Categoria \\
\hline $\begin{array}{l}\text { Saindo para divertir-se. } \\
\text { Gostando de estudar. } \\
\text { Praticando esportes. }\end{array}$ & $\begin{array}{l}\text { Usufruindo a adolescência } \\
\text { antes da gestação. }\end{array}$ & \\
\hline $\begin{array}{l}\text { Mudando tudo na vida. } \\
\text { Realizando adaptações na rotina. } \\
\text { Desejando um futuro promissor } \\
\text { para o filho. } \\
\text { Almejando o ingresso no nível } \\
\text { superior. } \\
\text { Planejando ingresso/retorno ao } \\
\text { trabalho. }\end{array}$ & $\begin{array}{l}\text { Descobrindo a rotina e as } \\
\text { adaptações de cuidados. } \\
\text { Almejando planos para o } \\
\text { futuro. }\end{array}$ & $\begin{array}{l}\text { Eventos para } \\
\text { cuidar de si: } \\
\text { gerando formas } \\
\text { de enfrentamento }\end{array}$ \\
\hline
\end{tabular}

Assim, as categorias foram construídas e refinadas, buscando o alcance da variação (Santos et al., 2021). Esses foram necessários para o processo de amostragem teórica e alcance da saturação das categorias, que ocorre quando novos dados coletados não são traduzidos em novas propriedades das categorias centrais ou insights teóricos (Charmaz, 2009). Foram construídas cinco categorias centrais e vinte subcategorias. Não foi utilizado software para gerenciamento dos dados (Santos et al., 2021).

\subsection{Aspectos Éticos do Estudo}

A presente investigação obteve anuência prévia do gestor municipal de saúde do município estudado e aprovação pelo Comitê de Ética em Pesquisa, no ano de 2017, parecer 2.069.288, protocolo CAAE: 66287317.0.0000.5393.

Seguindo diretrizes e normas regulamentadoras sobre pesquisa envolvendo seres humanos de acordo com a Resolução ํㅡ 466/12 do Conselho Nacional de Saúde, utilizouse um Termo de Consentimento Livre e Esclarecido (TCLE) para os representantes legais e um Termo de Assentimento Livre e Esclarecido (TALE) para as adolescentes menores de dezoito anos, e, para as adolescentes com dezoito anos de idade utilizou-se um TCLE (Santos et al., 2021).

$\mathrm{Na}$ condução das entrevistas, foi realizado um convite para a participação das mães adolescentes, com explicitação dos objetivos da pesquisa para elas e para os seus representantes legais (Santos et al., 2021). Nos casos em que a adolescente com idade inferior a 18 anos não convivia com os pais, antes da realização da entrevista a primeira autora realizou contato com eles buscando o consentimento, e, na impossibilidade de localização desse representante legal, a entrevista não foi realizada (Santos et al., 2021). 


\section{Resultados}

Após a análise do material qualitativo, iniciou-se o processo de elaboração da proposta de ferramenta EHC. As categorias centrais construídas no processo de análise foram utilizadas para a elaboração dos domínios do EHC, sendo: Eventos para cuidar de si: gerando formas de enfrentamento; Eventos para cuidar do outro: aprendendo com o cotidiano; Eventos da rede de cuidados: constituindo e fortalecendo diversos apoios; Eventos vulneráveis: enfrentando lacunas e fragilidades na atenção à saúde; e Eventos de sentido: suportando diferentes sentimentos e emoções (Santos et al., 2021).

$\mathrm{Na}$ elaboração da proposta de ferramenta, os domínios do EHC foram listados horizontalmente nas linhas e construídos por meio da análise das falas das mães adolescentes que apontaram aspectos sobre o cuidado de si e o cuidado da criança, bem como os fatores envolvidos nos processos de cuidado, com destaque para a rede de apoio, as vulnerabilidades, as situações e emoções vivenciadas (Santos et al., 2021).

A proposta de EHC apresenta dez domínios relacionados ao autocuidado da mãe adolescente e cinco domínios referentes ao cuidado da criança. Com relação ao autocuidado, os domínios abarcam aspectos referentes a idade, escolaridade, ocupação, atividades diárias/uso do tempo, relacionamentos, estilo de vida, sentimentos/preocupações, preconceito/discriminação por ser mãe adolescente, cuidados em saúde da mulher e planos para o futuro. Sobre o cuidado da criança, os domínios denotam aspectos concernentes às fontes de cuidado da criança, rotina da criança, crescimento da criança, desenvolvimento da criança e ações preventivas. Assim, os domínios dispostos na proposta de EHC buscam auxiliar o enfermeiro no levantamento de dados retrospectivos da vida da mãe adolescente, referentes aos eventos imbricados no processo do cuidado de si e do outro.

Dando prosseguimento ao processo de elaboração, inicialmente foram listados os domínios sobre o autocuidado, seguidos pelos domínios sobre o cuidado da criança. $\mathrm{Na}$ estrutura do EHC, para demarcá-los, foi destacada uma linha divisória, tornando-a mais espessa. Estes domínios representam informações consideradas importantes para os objetivos desta proposta de ferramenta, traduzidas em atividades, comportamentos ou eventos (Martyn \& Belli, 2002). Os domínios foram listados em uma sequência para facilitar a recordação, conforme recomendado por Martyn e Belli (2002), sendo que os três primeiros domínios incluem idade, escola e ocupação da mãe adolescente. Assim, os domínios foram construídos por meio da identificação e da compreensão de eventos entrelaçados no processo de autocuidado e de cuidado da criança, que permeiam a dinâmica de vida da adolescente mãe (Santos et al., 2021).

\section{Discussão}

O processo de construção dos domínios da ferramenta EHC para mães adolescentes denotam a utilização de elementos metodológicos da TFD construtivista associados com a técnica do snowball, que possibilitaram a identificação de mães adolescentes que vivenciavam diferentes cenários e a compreensão aprofundada de eventos e experiências relacionados à maternidade nessa fase da vida.

O entendimento é que a TFD se encontra aberta ao que ocorre em uma área substantiva, reconhecendo e nomeando os padrões de comportamento por meio de conceitos (Andrews et al., 2017). Para a TFD, os resultados não são imutáveis (Andrade et al., 2015) e o processo embasado nela não busca ser conclusivo, estando aberto a novas reformulações (Gabatz et al., 2017).

No processo de análise, os dados coletados foram submetidos a questionamentos constantes, conduzindo a produção de novos dados (Santos et al., 2018). Na presente pesquisa, foram constituídos três grupos amostrais, compostos por participantes diferentes que apresentavam experiências e vivências relevantes para compreensão do objeto de estudo (Santos et al., 2018). A composição de grupos amostrais apareceu como uma das estratégias para o alcance da amostragem teórica (Santos et al., 2018). 
A codificação possibilitou a separação e a conceituação dos dados (Santos et al., 2018). Por meio da codificação inicial linha a linha e da codificação focalizada, os dados oriundos das entrevistas com as mães adolescentes foram organizados e analisados de uma forma detalhada, em um constante movimento de questionamento e comparação. Esse movimento do pensar comparativamente (Solano et al., 2018) possibilitou reflexões, indagações, reconhecimentos de interligações e aprendizados.

No processo de análise também foram elaborados memorandos e construídos diagramas. Os memorandos, ao traduzirem as reflexões do pesquisador oriundas da análise e comparação constante dos dados coletados, favorecem a ilustração do processo de desenvolvimento de ideias e códigos (Santos et al., 2018). E os diagramas, ao apoiar o estabelecimento de relações conceituais, contribuem na construção de conceitos e hipóteses (Santos et al., 2016).

Assim, na pesquisa qualitativa em enfermagem, a TFD aparece como recurso metodológico que pode instigar a construção do conhecimento (Solano et al., 2018). Ademais, a utilização de elementos da TFD pode oferecer caminhos para a construção de ferramentas para apoiar o cuidado de enfermagem contextualizado, como o EHC.

\section{Considerações Finais}

Este estudo qualitativo apresentou os elementos metodológicos da TFD construtivista que forneceram alicerces para a construção dos domínios da ferramenta EHC para mães adolescentes.

A ferramenta EHC para mães adolescentes pode apoiar o processo de cuidado do profissional enfermeiro, ao possibilitar um olhar ampliado e particularizado para a vivência da maternidade na adolescência, buscando a promoção da qualidade de vida da adolescente mãe e da criança.

Considerando que diferentes fatores culturais e sociais influenciam o ato de maternar na adolescência, o presente estudo apresenta limitações pela realização em uma realidade contextualizada. Outras pesquisas são interessantes para a validação da ferramenta com mães adolescentes e enfermeiros e para a avaliação de sua aplicabilidade nos serviços de saúde de APS.

\section{Agradecimentos}

Conselho Nacional de Desenvolvimento Científico e Tecnológico (CNPq), Brasil, processo no. 309762/2019-7.

\section{Referências}

Andrade, P. R., Ohara, C. V. S., Borba, R. I. H., \& Ribeiro, C. A. (2015). Facing the difficult experience even with support: the underage adolescent experiencing motherhood. Revista Gaúcha de Enfermagem, 36, 111-118. https://doi.org/10.1590/1983-1447.2015.esp.56751

Andrews, T., Mariano, G. J. S., Santos, J. L. G., Koerber-Timmons, K., \& Silva, F. H. (2017). The methodology of classic grounded theory: considerations on its application in nursing research. Texto \& Contexto - Enfermagem, 26, e1560017. http://dx.doi.org/10.1590/0104070720170001560017

Biernacki, P., \& Waldorf, D. (1981). Snowball sampling: problems and techniques of chain referral sampling. Sociological Methods \& Research, 10, 141-163. https://doi.org/10.1177/004912418101000205

Charmaz, K. (2009). A construção da teoria fundamentada: guia prático para análise qualitativa. Tradução Joice Elias Costa. Artmed. 
Gabatz, R. I. B., Schwartz, E., Milbrath, V. M., Zillmer, J. G. V., \& Neves, E. T. (2017). Attachment theory, symbolic interactionism and grounded theory: articulating reference frameworks for research. Texto \& Contexto - Enfermagem, 26, e1940017. https://doi.org/10.1590/010407072017001940017

Martyn, K. K., \& Belli, R. F. (2002). Retrospective data collection using event history calendars. Nursing Research, 51, 270-274. https://doi.org/10.1097/00006199-200207000-00008

Martyn, K. K., Darling-Fisher, C., Pardee, M., Ronis, D. L., Felicetti, I. L., \& Saftner, M. A. (2012) Improving sexual risk communication with adolescents using event history calendars. The Journal of School Nursing, 28, 108-115. https://doi.org/10.1177/1059840511426577

Munro-Kramer, M. L., Fava, N. M., Banerjee, T., Darling-Fisher, C. S., Pardee, M., Villarruel, A. M., \& Martyn, K. K. (2017). The effect of a youth-centered sexual risk event history calendar (SREHC) assessment on sexual risk attitudes, intentions, and behavior. Journal of Pediatric Health Care, 31, 302-313. https://doi.org/10.1016/j.pedhc.2016.09.004

Santos, J. L. G., Cunha, K. S., Adamy, E. K., Backes, M. T. S., Leite, J. L., \& Sousa, F. G. M. (2018). Data analysis: comparison between the different methodological perspectives of the Grounded Theory. Revista da Escola de Enfermagem da USP, 52, e03303. https://doi.org/10.1590/s1980-220x2017021803303

Santos, J. L. G., Erdmann, A. L., Sousa, F. G. M., Lanzoni, G. M. M., Melo, A. L. S. F., \& Leite, J. L. (2016). Methodological perspectives in the use of grounded theory in nursing and health research. Escola Anna Nery, 20, e20160056. http://dx.doi.org/10.5935/1414-8145.20160056

Santos, J. S., Andrade, R. D., Silva, M. A. I., \& Mello, D. F. (2020). Nurse to adolescent health communication process: approach to Event History Calendar. Revista Brasileira de Enfermagem, 73, e20180454. http://dx.doi.org/10.1590/0034-7167-2018-0454

Santos, J. S., Andrade, R. D., Silva, M. A. I., \& Mello, D. F. (2021). Strengthening self-care and child care of adolescent mothers through an event history calendar. Journal of Pediatric Nursing, 57, e23-e28. https://doi.org/10.1016/j.pedn.2020.09.013

Santos, J. S. (2018). Desenvolvimento de um Calendário Histórico de Eventos para o fortalecimento do cuidado infantil por mães adolescentes (Tese de doutorado, Escola de Enfermagem de Ribeirão Preto, Universidade de São Paulo).

Solano, L. C., Miranda, F. A. N., Enders, B. C., \& Sousa, F. G. M. (2018). By avenue: dialoging about Grounded Theory. Revista Enfermagem UERJ, 26, e28047. https://doi.org/10.12957/reuerj.2018.28047 